\title{
Riemann-Hilbert Problem Associated with the Fourth-Order Dispersive Nonlinear Schrödinger Equation in Optics and Magnetic Mechanics
}

\author{
Beibei Hu ${ }^{1,2}$ (D) $\cdot$ Ling Zhang $^{1} \cdot$ Qinghong $\mathrm{Li}^{1} \cdot$ Ning Zhang ${ }^{3}$
}

Received: 31 January 2021 / Accepted: 5 July 2021 / Published online: 17 August 2021

(C) The Author(s) 2021

\begin{abstract}
In this paper, by using Fokas method, we study the initial-boundary value problems (IBVPs) of the fourth-order dispersive nonlinear Schrödinger (FODNLS) equation on the half-line, which can simulate the nonlinear transmission and interaction of ultrashort pulses in the high-speed optical fiber transmission system, and can also describe the nonlinear spin excitation phenomenon of one-dimensional Heisenberg ferromagnetic chain with eight poles and dipole interaction. By discussing the eigenfunctions of Lax pair of FODNLS equation and analyzing symmetry of the scattering matrix, we get a matrix Riemann-Hilbert $(\mathrm{RH})$ problem from for the IBVPs of FODNLS equation. Moreover, we get the potential function solution $u(x, t)$ of the FODNLS equation by solving this matrix RH problem. In addition, we also obtain that some spectral functions satisfy an important global relation.
\end{abstract}

Keywords Lax pair · Riemann-Hilbert problem · Fourth-order dispersive nonlinear Schrödinger equation · Initial-boundary value problems $\cdot$ Fokas method

Mathematics Subject Classification $35 \mathrm{G} 31 \cdot 35 \mathrm{Q} 15 \cdot 37 \mathrm{~K} 10 \cdot 45 \mathrm{D} 05$

Beibei Hu

hubsquare@chzu.edu.cn

$\bowtie$ Ling Zhang

originzhang@chzu.edu.cn

1 School of Mathematics and Finance, Chuzhou University, Chuzhou 239000, Anhui, People's Republic of China

2 Department of Physics, Zhejiang Normal University, Jinhua 321004, People's Republic of China

3 Department of Basical Courses, Shandong University of Science and Technology, Taian 271019, People's Republic of China 


\section{Introduction}

For a long time, finding the solutions of integrable equations has been a very important research topic in theory and application. The expression of exact solutions and some special solutions about integrable equations can provide important guidelines for the analysis of theirs various properties. However, there is no unified method to solve all integrable equations. With the in-depth study of integrable systems by scholars, a series of methods to solve the classic integrable equations have emerged, such as, inverse scattering transform (IST) method [1], Hirota method [2], Bäcklund transform [3], Darboux transform (DT) [4] and so on [5]. Among them, the IST method is the main analytical method for the exact solution of nonlinear integrable systems. However, due to the IST method is suitable for the limitations of the initial value conditions at infinity, it is almost only used to study the pure initial value problem of integrable equations. About many real-world phenomena and some studies in the fluctuation process, not only the initial value conditions need to be considered, but the boundary value conditions also need to be considered. Naturally, people need to replace the initial value problems with the initial-boundary value problems (IBVPs) in the research process.

In 1997, Fokas proposed a unified transformation method from the initial value problem to the IBVPs based on the IST method idea. This method can be used to investigate IBVPs of partial differential equation [6]. In the past 24 years, IBVPs of some classical integrable equations have be discussed via the Fokas method. For example, the modified Korteweg-de Vries (MKdV) equation [7], the nonlinear Schrödinger (NLS) equation [8], the Kaup-Newell equation [9], the stationary axisymmetric Einstein equations [10], the Ablowitz-Ladik system [11], the Kundu-Eckhaus equation [12], the Hirota equation [13, 14] and other equations [15-18]. In 2012, Lenells extended the Fokas method to the integrable equation with higher-order matrix spectrum, he proposed a more general unified transformation approach to solve IBVPs of integrable models [19] and he used the unified transformation approach to analyze IBVPs of Degasperis-Procesi equation [20]. After that, more and more researchers began to study the IBVPs of integrable model with higher-order matrix spectrum [21-28]. In particular, the long-time asymptotic behavior of the solution of the integrable systems were discussed by the nonlinear steepest descent method proposed by Deift and Zhou [29], see for instance the cases of the three-component coupled mKdV system [30] and the three-component coupled NLS system [31].

In this paper, our work is related to the fourth-order dispersive NLS (FODNLS) equation $[32,33]$

$$
\begin{aligned}
i u_{t}+\alpha_{1} u_{x x}+\alpha_{2} u|u|^{2}+\frac{\varepsilon^{2}}{12}\left(\alpha_{3} u_{x x x x}+\alpha_{4}|u|^{2} u_{x x}\right. \\
\left.+\alpha_{5} u^{2} \bar{u}_{x x}+\alpha_{6} u_{x}^{2} \bar{u}+\alpha_{7} u\left|u_{x}\right|^{2}+\alpha_{8}|u|^{4} u\right)=0
\end{aligned}
$$


where $u$ represents the amplitude of the slowly varying envelope of the wave, $x$ and $t$ are the normalized space and time variables, $\varepsilon^{2}$ is an infinitesimal dimensionless parameter representing the high-order linear and nonlinear strength, and $\alpha_{j}(j=1,2, \ldots, 8)$ is the actual parameter. The Eq. (1.1) is mainly derived from fiber optics and magnetism. On the one hand, in optics, Eq. (1.1) can simulate the nonlinear propagation and interaction of ultrashort pulses in high-speed fiber-optic transmission systems [34]. On the other hand, in magnetic mechanics, Eq. (1.1) can be used to describe the nonlinear spin excitation of a one-dimensional Heisenberg ferromagnetic chain with octuple and dipole interactions [35]. In particular, when the parameter value is $\alpha_{1}=\alpha_{3}=1, \alpha_{2}=\alpha_{5}=2, \alpha_{4}=8, \alpha_{6}=\alpha_{8}=6, \alpha_{7}=4$, and $\gamma=\frac{\varepsilon^{2}}{12}$, Eq. (1.1) becomes to

$$
i u_{t}+u_{x x}+2 u|u|^{2}+\gamma\left(u_{x x x x}+8|u|^{2} u_{x x}+2 u^{2} \bar{u}_{x x}+6 u_{x}^{2} \bar{u}+4 u\left|u_{x}\right|^{2}+6|u|^{4} u\right)=0,
$$

which is an integrable model, and many properties have been widely studied, such as, the Lax pair, the infinite conservation laws [36], the breather solution, and the higher-order rogue wave solution based on the DT method [37-39], the multi-soliton solutions through Riemann-Hilbert (RH) approach [40], the dark and bright solitary waves and rogue wave solution through phase plane analysis method [41], the bilinear form and the $\mathrm{N}$-soliton solution via the Hirota approach [42, 43]. However, as far as we know, the FODNLS equation (1.2) on the half-line has not been studied. In this paper, we utilize the Fokas method to discuss the IBVPs of the FODNLS equation (1.2) on the half-line domain $\Omega=\{(x, t): 0<x<\infty, 0<t<T\}$.

The paper is organized as follows. In Sect. 2, the eigenfunction for spectral analysis of the Lax pair are introduced. In Sect. 3, the essential functions $y(\zeta), z(\zeta), Y(\zeta), Z(\zeta)$ are further discussed. In Sect. 4, an important theorem is proposed. And the last section is devoted to conclusions.

\section{The spectral analysis}

Based on Ablowitz-Kaup-Newell-Segur scheme, the Lax pair of Eq. (1.2) is expressed as [36-40]

$$
\begin{gathered}
\Psi_{x}=(-i \zeta \Lambda+P) \Psi \\
\Psi_{t}=\left[\left(8 i \gamma \zeta^{4}-2 i \zeta^{2}\right) \Lambda-8 \gamma \zeta^{3} P-4 i \gamma \zeta^{2} A_{1}-2 \zeta A_{2}+i A_{3}\right] \Psi
\end{gathered}
$$

where $\zeta$ is a complex spectral parameter, $\Psi=\left(\Psi_{1}, \Psi_{2}\right)^{T}$ is the vector eigenfunction, the $2 \times 2$ matrices $\Lambda=\operatorname{diag}\{1,-1\}$, and $P, A_{1}, A_{2}$ and $A_{3}$ are defined by 


$$
\begin{aligned}
& P=\left(\begin{array}{cc}
0 & u \\
-\bar{u} & 0
\end{array}\right), A_{1}=\left(\begin{array}{cc}
|u|^{2} & u_{x} \\
\bar{u}_{x} & -|u|^{2}
\end{array}\right), \\
& A_{2}=\left(\begin{array}{cc}
\gamma\left(u \bar{u}_{x}-\bar{u} u_{x}\right) & -\gamma u_{x x}-\left(2 \gamma|u|^{2}+1\right) u \\
-\gamma \bar{u}_{x x}-\left(2 \gamma|u|^{2}+1\right) \bar{u} & -\gamma\left(u \bar{u}_{x}-\bar{u} u_{x}\right)
\end{array}\right) \text {, } \\
& A_{3}=\left(\begin{array}{cc}
A_{3}^{(11)} & \gamma u_{x x x}+\left(6 \gamma|u|^{2}+1\right) u_{x} \\
\gamma \bar{u}_{x x x}+\left(6 \gamma|u|^{2}+1\right) \bar{u}_{x} & -A_{3}^{(11)}
\end{array}\right) \text {, }
\end{aligned}
$$

with $A_{3}^{(11)}=\gamma\left(3|u|^{4}-\left|u_{x}\right|^{2}+\bar{u} u_{x x}+u \bar{u}_{x x}\right)+|u|^{2}$.

\subsection{The exact one-form}

The Lax pair Eqs. (2.1a)-(2.1b) are rewritten as

$$
\begin{gathered}
\Psi_{x}+i \zeta \Lambda \Psi=P(x, t, \zeta) \Psi, \\
\Psi_{t}-\left(8 i \gamma \zeta^{4}-2 i \zeta^{2}\right) \Lambda \Psi=R(x, t, \zeta) \Psi,
\end{gathered}
$$

where

$$
\begin{aligned}
R(x, t, \zeta)= & -8 \gamma \zeta^{3} P-4 i \gamma \zeta^{2} A_{1}-2 \zeta A_{2}+i A_{3} \\
= & -8 \gamma \zeta^{3} P+4 i \gamma \zeta^{2}\left(P^{2}+P_{x}\right) \Lambda+2 \gamma \zeta\left(P P_{x}-P_{x} P-P_{x x}+2 P^{3}\right) \Lambda \\
& -2 \zeta P \Lambda+i \gamma\left(3 P^{4}+P_{x}^{2}-P_{x x} P-P P_{x x}-P_{x x x}+6 P^{2} P_{x}-P_{x}\right) \Lambda-i P^{2} .
\end{aligned}
$$

Introducing the following function transformation

$$
\Psi(x, t, \zeta)=G(x, t, \zeta) e^{i\left[\left(8 \gamma \zeta^{4}-2 \zeta^{2}\right) t-\zeta x\right] \Lambda}, \quad 0<x<\infty, 0<t<T,
$$

we get

$$
\begin{gathered}
G_{x}+i \zeta[\Lambda, G]=P G, \\
G_{t}-i\left(8 \gamma \zeta^{4}-2 \zeta^{2}\right)[\Lambda, G]=R G,
\end{gathered}
$$

which can be expressed as the following full differential form

$$
d\left(e^{i\left[\zeta x-\left(8 \gamma \zeta^{4}-2 \zeta^{2}\right) t\right] \hat{\Lambda}} G(x, t, \zeta)\right)=F(x, t, \zeta),
$$

where exact one-form $F(x, t, \zeta)$ is

$$
F(x, t, \zeta)=e^{i\left[\zeta x-\left(8 \gamma \zeta^{4}-2 \zeta^{2}\right) t\right] \hat{\Lambda}}(P(x, t, \zeta) d x+R(x, t, \zeta) d t) G(x, t, \zeta),
$$

and $\hat{\Lambda}$ represents a matrix operator acting on a second order matrix $\Lambda$, i.e. $\hat{\Lambda} P=[\Lambda, P]$ and $e^{\hat{\Lambda}} P=e^{\Lambda} P e^{-\Lambda}$. 


\subsection{The Analytic and Bounded Eigenfunctions}

We assume that $u(x, t) \in \mathscr{S}$ with $(x, t) \in \Omega=\{(x, t): 0<x<\infty, 0<t<T\}$, and define eigenfunctions $\left\{G_{j}(x, t, \zeta)\right\}_{1}^{3}$ of Eqs. (2.5a)-(2.5b) as follows

$$
G_{j}(x, t, \zeta)=\mathrm{I}+\int_{\left(x_{j}, t_{j}\right)}^{(x, t)} e^{i\left[\left(8 \gamma \zeta^{4}-2 \zeta^{2}\right) t-\zeta x\right] \hat{\Lambda}} F(\xi, \tau, \zeta),
$$

where the integration path is $\left(x_{j}, t_{j}\right) \rightarrow(x, t)$, which is a directed smooth curves. It follows from the closed of the exact one-form that the integral of Eq. (1.2) is independent of the integration path. Therefore, one can choose three integral curve parallel to the axis shown in Fig. 1.

We might as well take $\left(x_{1}, t_{1}\right)=(0,0),\left(x_{2}, t_{2}\right)=(0, T)$, and $\left(x_{3}, t_{3}\right)=(\infty, t)$, then we have

$$
\begin{aligned}
& G_{1}(x, t, \zeta)= \mathrm{I}+\int_{0}^{x} e^{-i \zeta(x-\xi) \hat{\Lambda}}\left(P G_{1}\right)(\xi, t, \zeta) d \xi \\
&+e^{-i \zeta x \hat{\Lambda}} \int_{0}^{t} e^{i\left(8 \gamma \zeta^{4}-2 \zeta^{2}\right)(t-\tau) \hat{\Lambda}}\left(R_{1} G_{1}\right)(0, \tau, \Lambda) d \tau \\
& G_{2}(x, t, \zeta)=\mathrm{I}+\int_{0}^{x} e^{-i \zeta(x-\xi) \hat{\Lambda}}\left(P G_{2}\right)(\xi, t, \zeta) d \xi \\
&-e^{-i \zeta x \hat{\Lambda}} \int_{t}^{T} e^{i\left(8 \gamma \zeta^{4}-2 \zeta^{2}\right)(t-\tau) \hat{\Lambda}}\left(R_{1} G_{2}\right)(0, \tau, \Lambda) d \tau, \\
& G_{3}(x, t, \zeta)=\mathrm{I}-\int_{x}^{\infty} e^{-i \zeta(x-\xi) \hat{\Lambda}}\left(P G_{3}\right)(\xi, t, \zeta) d \xi .
\end{aligned}
$$

On the one hand, any point $(x, t)$ on the integral curves $\left\{\gamma_{j}\right\}_{1}^{3}$ satisfies the following inequalities

$$
\begin{aligned}
& \gamma_{1}: x-\xi \geq 0, t-\tau \geq 0, \\
& \gamma_{2}: x-\xi \geq 0, t-\tau \leq 0,
\end{aligned}
$$
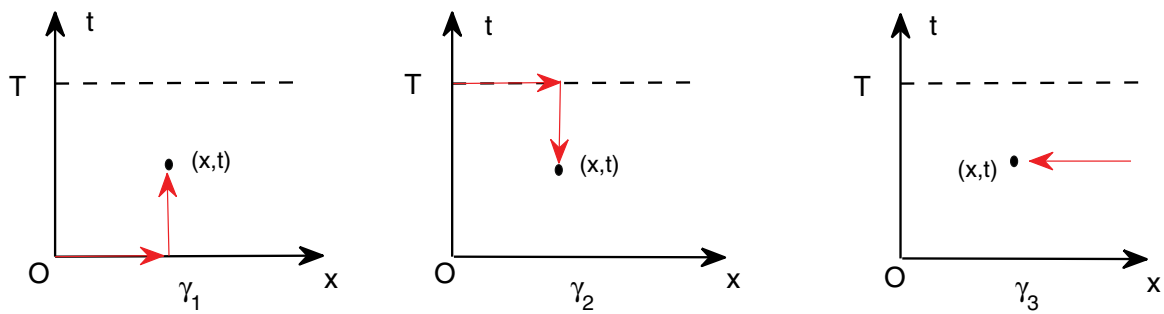

Fig. 1 The three contours $\gamma_{1}, \gamma_{2}, \gamma_{3}$ in the $(x, t)$-domain 


$$
\gamma_{3}: x-\xi \leq 0
$$

On the other hand, it follows from the Eq. (2.8) that the first column of $G_{j}(x, t, \zeta)$ contains $e^{2 i \zeta(x-\xi)-2 i\left(8 \gamma \zeta^{4}-2 \zeta^{2}\right)(t-\tau)}$. Thus, for $\zeta \in \mathrm{C}$, we can calculate the bounded analytic region of $\left[G_{j}(x, t, \zeta)\right]_{1}$, where $\zeta$ must satisfy

$$
\begin{aligned}
& {\left[G_{1}\right]_{1}(x, t, \zeta):\{\operatorname{Im} \zeta \geq 0\} \cap\left\{\operatorname{Im}\left(8 \gamma \zeta^{4}-2 \zeta^{2}\right) \geq 0\right\},} \\
& {\left[G_{2}\right]_{1}(x, t, \zeta):\{\operatorname{Im} \zeta \geq 0\} \cap\left\{\operatorname{Im}\left(8 \gamma \zeta^{4}-2 \zeta^{2}\right) \leq 0\right\},} \\
& {\left[G_{3}\right]_{1}(x, t, \zeta):\{\operatorname{Im} \zeta \leq 0\} .}
\end{aligned}
$$

Similarly, the second column of $G_{j}(x, t, \zeta)$ contains $e^{-2 i \zeta(x-\xi)+2 i\left(8 \gamma \zeta^{4}-2 \zeta^{2}\right)(t-\tau)}$. Then, for $\zeta \in \mathrm{C}$, we can also calculate the bounded analytic region of the eigenfunctions $\left[G_{j}(x, t, \zeta)\right]_{2}$, where $\zeta$ must satisfy

$$
\begin{aligned}
& {\left[G_{1}\right]_{2}(x, t, \zeta):\{\operatorname{Im} \zeta \leq 0\} \cap\left\{\operatorname{Im}\left(8 \gamma \zeta^{4}-2 \zeta^{2}\right) \leq 0\right\},} \\
& {\left[G_{2}\right]_{2}(x, t, \zeta):\{\operatorname{Im} \zeta \leq 0\} \cap\left\{\operatorname{Im}\left(8 \gamma \zeta^{4}-2 \zeta^{2}\right) \geq 0\right\}} \\
& {\left[G_{3}\right]_{2}(x, t, \zeta):\{\operatorname{Im} \zeta \geq 0\} .}
\end{aligned}
$$

Here the $\left[G_{j}\right]_{k}(x, t, \zeta)$ denotes the $k$-columns of $G_{j}(x, t, \zeta)$. By calculation, we get the bounded analytic region of $G_{j}(x, t, \zeta)$ as follows

$$
\begin{gathered}
G_{1}(x, t, \zeta)=\left(\left[G_{1}\right]_{1}^{L_{1} \cup L_{3}}(x, t, \zeta),\left[G_{1}\right]_{2}^{L_{6} \cup L_{8}}(x, t, \zeta)\right), \\
G_{2}(x, t, \zeta)=\left(\left[G_{2}\right]_{1}^{L_{2} \cup L_{4}}(x, t, \zeta),\left[G_{2}\right]_{2}^{L_{5} \cup L_{7}}(x, t, \zeta)\right), \\
G_{3}(x, t, \zeta)=\left(\left[G_{3}\right]_{1}^{L_{5} \cup L_{6} \cup L_{7} \cup L_{8}}(x, t, \zeta),\left[G_{3}\right]_{2}^{L_{1} \cup L_{2} \cup L_{3} \cup L_{4}}(x, t, \zeta)\right),
\end{gathered}
$$

where $G_{j}^{L_{i}}(x, t, \zeta)$ represents that the bounded analytic region of $\left\{G_{j}(x, t, \zeta)\right\}_{1}^{3}$ is $\zeta \in L_{i}, i=1,2, \ldots, 8$, and $L_{i}, i=1,2, \ldots, 8$ are shown in Fig. 2.

To establish the RH problem of the FODNLS Eq. (1.2), we define two special functions $\psi(\zeta)$ and $\phi(\zeta)$ with the eigenfunction $\left\{G_{j}(x, t, \zeta)\right\}_{1}^{3}$ as follows

$$
\begin{aligned}
& G_{3}(x, t, \zeta)=G_{1}(x, t, \zeta) e^{i\left[\left(8 \gamma \zeta^{4}-2 \zeta^{2}\right) t-\zeta x\right] \hat{\Lambda}} \psi(\zeta), \\
& G_{2}(x, t, \zeta)=G_{1}(x, t, \zeta) e^{i\left[\left(8 \gamma \zeta^{4}-2 \zeta^{2}\right) t-\zeta x\right] \hat{\Lambda}} \phi(\zeta) .
\end{aligned}
$$

Let $(x, t)=(0,0)$ in Eq. $(2.14 \mathrm{a})$, and let $(x, t)=(0, T)$ in Eq. $(2.14 \mathrm{~b})$, we obtain the following relationship 


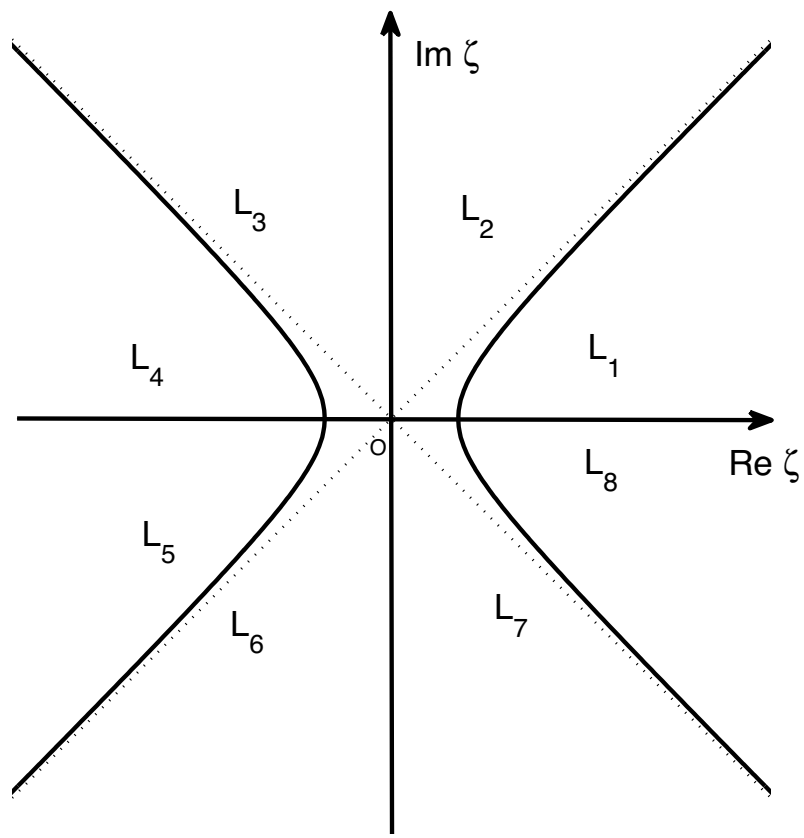

Fig. 2 The areas $L_{i}, i=1, \ldots, 8$ division on the complex $\zeta$ plane

$$
\psi(\zeta)=G_{3}(0,0, \zeta), \phi(\zeta)=G_{2}(0,0, \zeta)=\left[e^{i\left(8 \gamma \zeta^{4}-2 \zeta^{2}\right) T \hat{\Lambda}} G_{1}(0, T, \zeta)\right]^{-1}
$$

then, we get

$$
G_{3}(x, t, \zeta)=G_{1}(x, t, \zeta) e^{i\left[\left(8 \gamma \zeta^{4}-2 \zeta^{2}\right) t-\zeta x\right] \hat{\Lambda}} G_{3}(0,0, \zeta)
$$

and

$$
G_{2}(x, t, \zeta)=G_{1}(x, t, \zeta) e^{i\left[\left(8 \gamma \zeta^{4}-2 \zeta^{2}\right) t-\zeta x\right] \hat{\Lambda}}\left[e^{-i\left(8 \gamma \zeta^{4}-2 \zeta^{2}\right) T \hat{\Lambda}} G_{1}(0, T, \zeta)\right]^{-1},
$$

it follows from the Eqs. (2.16)-(2.17) that

$$
G_{2}(x, t, \zeta)=G_{3}(x, t, \zeta) e^{i\left[\left(8 \gamma \zeta^{4}-2 \zeta^{2}\right) t-\zeta x\right] \hat{\Lambda}}(\psi(\zeta))^{-1} \phi(\zeta) .
$$

Particularly, taking $x=0$ in the eigenfunction $G_{j}(x, t, \zeta), j=1,2$, we have

$$
\begin{aligned}
G_{1}(0, t, \zeta) & =\left(\left[G_{1}\right]_{1}^{L_{1} \cup L_{3} \cup L_{5} \cup L_{7}}(0, t, \zeta),\left[G_{1}\right]_{2}^{L_{2} \cup L_{4} \cup L_{6} \cup L_{8}}(0, t, \zeta)\right) \\
& =\mathrm{I}+\int_{0}^{t} e^{i\left(8 \gamma \zeta^{4}-2 \zeta^{2}\right)(t-\tau) \hat{\Lambda}}\left(R G_{1}\right)(0, \tau, \zeta) d \tau,
\end{aligned}
$$




$$
\begin{aligned}
G_{2}(0, t, \zeta) & =\left(\left[G_{2}\right]_{1}^{L_{2} \cup L_{4} \cup L_{6} \cup L_{8}}(0, t, \zeta),\left[G_{2}\right]_{2}^{L_{1} \cup L_{3} \cup L_{5} \cup L_{7}}(0, t, \zeta)\right) \\
& =\mathrm{I}-\int_{t}^{T} e^{i\left(8 \gamma \zeta^{4}-2 \zeta^{2}\right)(t-\tau) \hat{\Lambda}}\left(R G_{2}\right)(0, \tau, \zeta) d \tau
\end{aligned}
$$

and when $t=0$ in the eigenfunction $G_{1}(x, t, \zeta), G_{3}(x, t, \zeta)$, we have

$$
\begin{aligned}
G_{1}(x, 0 ; \zeta) & =\left(\left[G_{1}\right]_{1}^{L_{1} \cup L_{2} \cup L_{3} \cup L_{4}}(x, 0, \zeta),\left[G_{1}\right]_{2}^{L_{5} \cup L_{6} \cup L_{7} \cup L_{8}}(x, 0, \zeta)\right) \\
& =\mathrm{I}+\int_{0}^{x} e^{-i \zeta(x-\xi) \hat{\Lambda}}\left(P G_{1}\right)(\xi, 0, \zeta) d \xi \\
G_{3}(x, 0 ; \zeta) & =\left(\left[G_{3}\right]_{1}^{L_{5} \cup L_{6} \cup L_{7} \cup L_{8}}(z, 0, \zeta),\left[G_{3}\right]_{2}^{L_{1} \cup L_{2} \cup L_{3} \cup L_{4}}(z, 0, \zeta)\right) \\
& =\mathrm{I}-\int_{x}^{\infty} e^{-i \zeta(x-\xi) \hat{\Lambda}}\left(P G_{3}\right)(\xi, 0, \zeta) d \xi
\end{aligned}
$$

Assume that $u_{0}(x)=u(x, t=0)$ is an initial data of the functions $u(x, t)$, and $v_{0}(t)=u(x=0, t), \quad v_{1}(t)=u_{x}(x=0, t), \quad v_{2}(t)=u_{x x}(x=0, t), \quad v_{3}(t)=u_{x x x}(x=0, t)$ be boundary data of the functions $u_{x}(x, t), u_{x x}(x, t), u_{x x x}(x, t)$. Then, the matrices $P(x, 0, \zeta)$ and $R(0, t, \zeta)$ have the following form

$$
P(x, 0, \zeta)=\left(\begin{array}{cc}
0 & u_{0} \\
-\bar{u}_{0} & 0
\end{array}\right), R(0, t, \zeta)=\left(\begin{array}{cc}
R_{11}(0, t, \zeta) & R_{12}(0, t, \zeta) \\
R_{21}(0, t, \zeta) & -R_{11}(0, t, \zeta)
\end{array}\right)
$$

with

$$
\begin{aligned}
& R_{11}(0, t, \zeta)=-4 i \gamma \zeta^{2} v_{0}^{2}-2 \gamma \zeta\left(v_{0} \bar{v}_{1}-v_{1} \bar{v}_{0}\right)+i \gamma\left(3\left|v_{0}\right|^{4}-\left|v_{1}\right|^{2}+\bar{v}_{0} v_{2}+v_{0} \bar{v}_{2}\right)+i\left|v_{0}\right|^{2}, \\
& R_{12}(0, t, \zeta)=-8 \gamma \zeta^{3} v_{0}-4 i \gamma \zeta^{2} v_{1}+2 \gamma \zeta v_{2}+2 \zeta\left(2 \gamma\left|v_{0}\right|^{2}+1\right) v_{0}+i \gamma v_{3}+i\left(6 \gamma\left|v_{0}\right|^{2}+1\right) v_{1}, \\
& R_{21}(0, t, \zeta)=-8 \gamma \zeta^{3} \bar{v}_{0}-4 i \gamma \zeta^{2} \bar{v}_{1}+2 \gamma \zeta \bar{v}_{2}+2 \zeta\left(2 \gamma\left|v_{0}\right|^{2}+1\right) \bar{v}_{0}+i \gamma \bar{v}_{3}+i\left(6 \gamma\left|v_{0}\right|^{2}+1\right) \bar{v}_{1}
\end{aligned}
$$

\subsection{The Other Properties of the Eigenfunctions}

Proposition 2.1 The matrix-valued functions $G_{j}(x, t, \zeta)=\left(\left[G_{j}\right]_{1}(x, t, \zeta),\left[G_{j}\right]_{2}(x, t, \zeta)\right)$ $(j=1,2,3)$ given in Eq. (2.8) possess the following analytical properties:

- $\operatorname{det} G_{j}(x, t, \zeta)=1$;

- The $\left[G_{1}\right]_{1}(x, t, \zeta)$ is an analytic function for $\zeta \in L_{1} \cup L_{3}$, and the $\left[G_{1}\right]_{2}(x, t, \zeta)$ is also an analytic function for $\zeta \in L_{6} \cup L_{8}$;

- The $\left[G_{2}\right]_{1}(x, t, \zeta)$ is an analytic function for $\zeta \in L_{2} \cup L_{4}$, and the $\left[G_{2}\right]_{2}(x, t, \zeta)$ is also an analytic function for $\zeta \in L_{5} \cup L_{7}$;

- The $\left[G_{3}\right]_{1}(x, t, \zeta)$ is an analytic function for $\zeta \in L_{5} \cup L_{6} \cup L_{7} \cup L_{8}$, and the $\left[G_{3}\right]_{2}(x, t, \zeta)$ is also an analytic function for $\zeta \in L_{1} \cup L_{2} \cup L_{3} \cup L_{4}$;

- The $\left[G_{j}\right]_{1}(x, t, \zeta) \rightarrow(1,0)^{T}$ and $\left[G_{j}\right]_{2}(x, t, \zeta) \rightarrow(0,1)^{T}$, as $\zeta \rightarrow \infty$. 
Proposition 2.2 Indeed, $\psi(\zeta), \phi(\zeta)$ defined in Eqs. (2.14a)-(2.14b) or Eq. (2.15) are expressed as

$$
\begin{gathered}
\psi(\zeta)=\mathrm{I}-\int_{0}^{\infty} e^{i \zeta \xi \hat{\Lambda}}\left(P G_{3}\right)(\xi, 0, \zeta) d \xi \\
\phi^{-1}(\zeta)=\mathrm{I}+\int_{0}^{T} e^{i\left(8 \gamma \zeta^{4}-2 \zeta^{2}\right) \tau \hat{\Lambda}}\left(R G_{1}\right)(0, \tau, \zeta) d \tau .
\end{gathered}
$$

It follows from the symmetry properties of $P(x, t, \zeta)$ and $R(x, t, \zeta)$ that

$$
\left(G_{j}(x, t, \zeta)\right)_{11}=\overline{\left(G_{j}(x, t, \bar{\zeta})\right)_{11}}, \quad\left(G_{j}(x, t, \zeta)\right)_{21}=-\overline{\left(G_{j}(x, t, \bar{\zeta})\right)_{12}},
$$

then we have

$$
\begin{array}{ll}
\psi_{11}(\zeta)=\overline{\psi_{11}(\bar{\zeta})}, & \psi_{21}(\zeta)=-\overline{\psi_{12}(\bar{\zeta})} \\
\phi_{11}(\zeta)=\overline{\phi_{11(\bar{\zeta})}}, & \phi_{21}(\zeta)=-\overline{\phi_{12}(\bar{\zeta})}
\end{array}
$$

Assume that the $\psi(\zeta)$ and $\phi(\zeta)$ admit the matrix form as follows

$$
\psi(\zeta)=\left(\begin{array}{cc}
\overline{y(\bar{\zeta})} & z(\zeta) \\
-\overline{z(\bar{\zeta})} & y(\zeta)
\end{array}\right), \phi(\zeta)=\left(\begin{array}{cc}
\overline{Y(\bar{\zeta})} & Z(\zeta) \\
-\overline{Z(\bar{\zeta})} & Y(\zeta)
\end{array}\right)
$$

In terms of the Eq. (2.15) and Eqs. (2.22a)-(2.22b), we know that the following properties are true.

$$
\text { - }\left(\begin{array}{l}
z(\zeta) \\
y(\zeta)
\end{array}\right)=\left[G_{3}\right]_{2}^{L_{1} \cup L_{2} \cup L_{3} \cup L_{4}}(0,0, \zeta)=\left(\begin{array}{c}
\left(G_{3}\right)_{12}^{L_{1} \cup L_{2} \cup L_{3} \cup L_{4}}(0,0, \zeta) \\
\left(G_{3}\right)_{22}^{L_{1} \cup L_{2} \cup L_{3} \cup L_{4}}(0,0, \zeta)
\end{array}\right)
$$

where the vector function $\left[G_{3}\right]_{2}^{L_{1} \cup L_{2} \cup L_{3} \cup L_{4}}(x, 0, \zeta)$ satisfies the ordinary differential equation as follows

$$
\begin{gathered}
\partial_{x}\left[G_{3}\right]_{2}^{L_{1} \cup L_{2} \cup L_{3} \cup L_{4}}(x, 0, \zeta)+2 i \zeta\left(\begin{array}{ll}
1 & 0 \\
0 & 0
\end{array}\right)\left[G_{3}\right]_{2}^{L_{1} \cup L_{2} \cup L_{3} \cup L_{4}}(x, 0, \zeta) \\
=P(x, 0, \zeta)\left[G_{3}\right]_{2}^{L_{1} \cup L_{2} \cup L_{3} \cup L_{4}}(x, 0, \zeta), 0<x<\infty,
\end{gathered}
$$

here $P(x, 0, \zeta)$ is given in Eq. (2.21) and

$$
\lim _{x \rightarrow \infty}\left[G_{3}\right]_{2}^{L_{1} \cup L_{2} \cup L_{3} \cup L_{4}}(x, 0, \zeta)=(0,1)^{T} .
$$




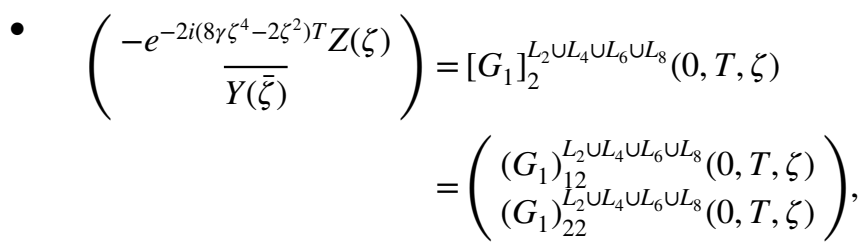

where the vector function $\left[G_{1}\right]_{2}^{L_{2} \cup L_{4} \cup L_{6} \cup L_{8}}(0, t, \zeta)$ satisfies the ordinary differential equation as follows

$$
\begin{gathered}
\partial_{t}\left[G_{1}\right]_{2}^{L_{2} \cup L_{4} \cup L_{6} \cup L_{8}}(0, t, \zeta)-2 i\left(8 \gamma \zeta^{4}-2 \zeta^{2}\right)\left(\begin{array}{ll}
1 & 0 \\
0 & 0
\end{array}\right)\left[G_{1}\right]_{2}^{L_{2} \cup L_{4} \cup L_{6} \cup L_{8}}(0, t, \zeta) \\
=R(0, t, \zeta)\left[G_{1}\right]_{2}^{L_{2} \cup L_{4} \cup L_{6} \cup L_{8}}(0, t, \zeta), 0<t<T,
\end{gathered}
$$

here $R(0, t, \zeta)$ is given in Eq. (2.21) and

$$
\left[G_{1}\right]_{2}^{L_{2} \cup L_{4} \cup L_{6} \cup L_{8}}(0,0, \zeta)=(0,1)^{T} .
$$

- $y(-\zeta)=y(\zeta), z(-\zeta)=-z(\zeta), \quad Y(-\zeta)=Y(\zeta), Z(-\zeta)=-Z(\zeta)$

For $\zeta \in \mathrm{R}, \operatorname{det} \psi(\zeta)=|y(\zeta)|^{2}+|z(\zeta)|^{2}=1$.

- For $\zeta \in \mathrm{C}, \operatorname{det} \phi(\zeta)=Y(\zeta) \overline{Y(\bar{\zeta})}+Z(\zeta) \overline{Z(\bar{\zeta})}=1,\left(\zeta \in \gamma_{m}\right.$, if $\left.T=\infty\right)$, where curve $\gamma_{m}, m=1,2,3,4$ are given in Eq. (2.30).

$$
\begin{aligned}
& y(\zeta)=1+O\left(\frac{1}{\zeta}\right), \quad z(\zeta)=O\left(\frac{1}{\zeta}\right), \text { as } \zeta \rightarrow \infty, \\
& Y(\zeta)=1+O\left(\frac{1}{\zeta}\right)+O\left(\frac{e^{-2 i\left(8 \gamma \zeta^{4}-2 \zeta^{2}\right) T}}{\zeta}\right), \\
& Z(\zeta)=O\left(\frac{1}{\zeta}\right)+O\left(\frac{e^{-2 i\left(8 \gamma \zeta^{4}-2 \zeta^{2}\right) T}}{\zeta}\right), \text { as } \zeta \rightarrow \infty
\end{aligned}
$$

\subsection{The Basic Riemann-Hilbert Problem}

In order to facilitate calculation, we introduce the symbolic assumptions as follows

$$
\begin{gathered}
\omega(x, t, \zeta)=\zeta x-\left(8 \gamma \zeta^{4}-2 \zeta^{2}\right) t \\
\rho(\zeta)=y(\zeta) \overline{Y(\bar{\zeta})}+z(\zeta) \overline{Z(\bar{\zeta})} \\
\kappa(\zeta)=\overline{y(\bar{\zeta}) Z(\bar{\zeta})}-\overline{z(\bar{\zeta}) Y(\bar{\zeta})}
\end{gathered}
$$




$$
\delta(\zeta)=\frac{z(\zeta)}{\overline{y(\bar{\zeta})}}, \Delta(\zeta)=-\frac{\overline{Z(\bar{\zeta})}}{y(\zeta) \rho(\zeta)}
$$

then, we have

$$
\begin{aligned}
& \overline{Z(\bar{\zeta})}=y(\zeta) \kappa(\zeta)+\overline{z(\bar{\zeta})} \rho(\zeta), \\
& \rho(\zeta) \overline{\rho(\bar{\zeta})}-\kappa(\zeta \overline{\kappa(\bar{\zeta})}=1, \\
& \rho(\zeta)=1+O\left(\frac{1}{\zeta}\right), \kappa(\zeta)=O\left(\frac{1}{\zeta}\right) \text { as } \zeta \rightarrow \infty, \\
& \rho(-\zeta)=\rho(\zeta), \kappa(-\zeta)=-\kappa(\zeta),
\end{aligned}
$$

and the matrix function $D(x, t, \zeta)$ is defined by

$$
\begin{aligned}
& D_{+}(x, t, \zeta)=\left(\frac{\left[G_{1}\right]_{1}^{L_{1} \cup L_{3}}(x, t, \zeta)}{y(\zeta)},\left[G_{3}\right]_{2}^{L_{1} \cup L_{2} \cup L_{3} \cup L_{4}}(x, t, \zeta)\right), \zeta \in L_{1} \cup L_{3}, \\
& D_{-}(x, t, \zeta)=\left(\frac{\left[G_{2}\right]_{1}^{L_{2} \cup L_{4}}(x, t, \zeta)}{\rho(\zeta)},\left[G_{3}\right]_{2}^{L_{1} \cup L_{2} \cup L_{3} \cup L_{4}}(x, t, \zeta)\right), \zeta \in L_{2} \cup L_{4}, \\
& D_{+}(x, t, \zeta)=\left(\left[G_{3}\right]_{1}^{L_{5} \cup L_{6} \cup L_{7} \cup L_{8}}(x, t, \zeta), \frac{\left[G_{2}\right]_{2}^{L_{5} \cup L_{7}}(x, t, \zeta)}{\overline{\rho(\bar{\zeta})}}\right), \zeta \in L_{5} \cup L_{7}, \\
& D_{-}(x, t, \zeta)=\left(\left[G_{3}\right]_{1}^{L_{5} \cup L_{6} \cup L_{7} \cup L_{8}}(x, t, \zeta), \frac{\left[G_{1}\right]_{2}^{L_{6} \cup L_{8}}(x, t, \zeta)}{\overline{y(\bar{\zeta})}}\right), \zeta \in L_{6} \cup L_{8} .
\end{aligned}
$$

Obviously, the above definitions indicate that

$$
\operatorname{det} D(x, t, \zeta)=1, D(x, t, \zeta) \rightarrow \mathrm{I}, \text { as } \zeta \rightarrow \infty .
$$

Theorem 2.3 The matrix function $D(x, t, \zeta)$ defined by Eqs. (2.27a)-(2.27d) admits the jump relation on the curves $\Gamma_{m}, m=1, \ldots, 4$ as follows

$$
D_{+}(x, t, \zeta)=D_{-}(x, t, \zeta) Q(x, t, \zeta), \zeta \in \Gamma_{m}, m=1, \ldots, 4,
$$

where

$$
Q(x, t, \zeta)=\left\{\begin{array}{l}
Q_{1}(x, t, \zeta), \zeta \in \Gamma_{1} \doteq\left\{\bar{L}_{1} \cup \bar{L}_{3}\right\} \cap\left\{\bar{L}_{2} \cup \bar{L}_{4}\right\} \\
Q_{2}(x, t, \zeta)=Q_{3} Q_{4}^{-1} Q_{1}, \zeta \in \Gamma_{2} \doteq\left\{\bar{L}_{2} \cup \bar{L}_{4}\right\} \cap\left\{\bar{L}_{5} \cup \bar{L}_{7}\right\} \\
Q_{3}(x, t, \zeta), \zeta \in \Gamma_{3} \doteq\left\{\bar{L}_{5} \cup \bar{L}_{7}\right\} \cap\left\{\bar{L}_{6} \cup \bar{L}_{8}\right\} \\
Q_{4}(x, t, \zeta), \zeta \in \Gamma_{4} \doteq\left\{\bar{L}_{6} \cup \bar{L}_{8}\right\} \cap\left\{\bar{L}_{1} \cup \bar{L}_{3}\right\}
\end{array}\right.
$$


and

$$
\begin{aligned}
& Q_{1}(x, t, \zeta)=\left(\begin{array}{cc}
1 & 0 \\
\Delta(\zeta) e^{2 i \omega(\zeta)} & 1
\end{array}\right), \\
& Q_{2}(x, t, \zeta)=\left(\begin{array}{cc}
1-(\delta(\zeta)+\overline{\Delta(\bar{\zeta})})(\Delta(\zeta)+\overline{\delta(\bar{\zeta})}) & \left(\delta(\zeta)+\overline{\Delta(\bar{\zeta}))} e^{-2 i \omega(\zeta)}\right. \\
(\Delta(\zeta)+\overline{\delta(\bar{\zeta})}) e^{2 i \omega(\zeta)} & 1
\end{array}\right), \\
& Q_{3}(x, t, \zeta)=\left(\begin{array}{cc}
1 & \overline{\Delta(\bar{\zeta})} e^{-2 i \omega(\zeta)} \\
0 & 1
\end{array}\right) \\
& Q_{4}(x, t, \zeta)=\left(\begin{array}{cc}
1 & -\delta(\zeta) e^{-2 i \omega(\zeta)} \\
-\overline{\delta(\bar{\zeta})} e^{2 i \omega(\zeta)} & 1+|\delta(\zeta)|^{2}
\end{array}\right)
\end{aligned}
$$

Proof In terms of the Eqs. (2.14a)-(2.14b) and Eq. (2.23), we have

$$
\begin{aligned}
& \overline{y(\bar{\zeta})}\left[G_{1}\right]_{1}^{L_{1} \cup L_{3}}(x, t, \zeta)-\overline{z(\bar{\zeta})} e^{2 i \omega(\zeta)}\left[G_{1}\right]_{2}^{L_{6} \cup L_{8}}(x, t, \zeta)=\left[G_{3}\right]_{1}^{L_{5} \cup L_{6} \cup L_{7} \cup L_{8}}(x, t, \zeta), \\
& z(\zeta) e^{-2 i \omega(\zeta)}\left[G_{1}\right]_{1}^{L_{1} \cup L_{3}}(x, t, \zeta)+y(\zeta)\left[G_{1}\right]_{2}^{L_{6} \cup L_{8}}(x, t, \zeta)=\left[G_{3}\right]_{2}^{L_{1} \cup L_{2} \cup L_{3} \cup L_{4}}(x, t, \zeta),
\end{aligned}
$$

and

$$
\begin{aligned}
& \overline{Y(\bar{\zeta})}\left[G_{1}\right]_{1}^{L_{1} \cup L_{3}}(x, t, \zeta)-\overline{Z(\bar{\zeta})} e^{2 i \omega(\zeta)}\left[G_{1}\right]_{2}^{L_{6} \cup L_{8}}(x, t, \zeta)=\left[G_{2}\right]_{1}^{L_{2} \cup L_{4}}(x, t, \zeta) \\
& Z(\zeta) e^{-2 i \omega(\zeta)}\left[G_{1}\right]_{1}^{L_{1} \cup L_{3}}(x, t, \zeta)+Y(\zeta)\left[G_{1}\right]_{2}^{L_{6} \cup L_{8}}(x, t, \zeta)=\left[G_{2}\right]_{2}^{L_{5} \cup L_{7}}(x, t, \zeta)
\end{aligned}
$$

according to the Eqs. (2.31a)-(2.32b) and Eqs. (2.26a)-(2.26d), we get

$$
\begin{aligned}
& \rho(\zeta)\left[G_{3}\right]_{1}^{L_{5} \cup L_{6} \cup L_{7} \cup L_{8}}(x, t, \zeta)-\kappa(\zeta) e^{2 i \omega(\zeta)}\left[G_{3}\right]_{2}^{L_{1} \cup L_{2} \cup L_{3} \cup L_{4}}(x, t, \zeta)=\left[G_{2}\right]_{1}^{L_{2} \cup L_{4}}(x, t, \zeta), \\
& \overline{\kappa(\bar{\zeta})} e^{-2 i \omega(\zeta)}\left[G_{3}\right]_{1}^{L_{5} \cup L_{6} \cup L_{7} \cup L_{8}}(x, t, \zeta)+\overline{\rho(\bar{\zeta})}\left[G_{3}\right]_{2}^{L_{1} \cup L_{2} \cup L_{3} \cup L_{4}}(x, t, \zeta)=\left[G_{2}\right]_{2}^{L_{5} \cup L_{7}}(x, t, \zeta) .
\end{aligned}
$$

By the Eqs. (2.27a)-(2.27d) and Eq. (2.29), we have

$$
\begin{aligned}
& \left(\frac{\left[G_{1}\right]_{1}^{L_{1} \cup L_{3}}(x, t, \zeta)}{y(\zeta)},\left[G_{3}\right]_{2}^{L_{1} \cup L_{2} \cup L_{3} \cup L_{4}}(x, t, \zeta)\right) \\
& \quad=\left(\frac{\left[G_{2}\right]_{1}^{L_{2} \cup L_{4}}(x, t, \zeta)}{\rho(\zeta)},\left[G_{3}\right]_{2}^{L_{1} \cup L_{2} \cup L_{3} \cup L_{4}}(x, t, \zeta)\right) Q_{1}(x, t, \zeta),
\end{aligned}
$$




$$
\begin{aligned}
& \left(\left[G_{3}\right]_{1}^{L_{5} \cup L_{6} \cup L_{7} \cup L_{8}}(x, t, \zeta), \frac{\left[G_{2}\right]_{2}^{L_{5} \cup L_{7}}(x, t, \zeta)}{\overline{\rho(\bar{\zeta})}}\right) \\
& =\left(\frac{\left[G_{2}\right]_{1}^{L_{2} \cup L_{4}}(x, t, \zeta)}{\rho(\zeta)},\left[G_{3}\right]_{2}^{L_{1} \cup L_{2} \cup L_{3} \cup L_{4}}(x, t, \zeta)\right) Q_{2}(x, t, \zeta), \\
& \left(\left[G_{3}\right]_{1}^{L_{5} \cup L_{6} \cup L_{7} \cup L_{8}}(x, t, \zeta), \frac{\left[G_{2}\right]_{2}^{L_{5} \cup L_{7}(x, t, \zeta)}}{\overline{\rho(\bar{\zeta})}}\right) \\
& =\left(\left[G_{3}\right]_{1}^{L_{5} \cup L_{6} \cup L_{7} \cup L_{8}}(x, t, \zeta), \frac{\left[G_{1}\right]_{2}^{L_{6} \cup L_{8}}(x, t, \zeta)}{\overline{y(\bar{\zeta})}}\right) Q_{3}(x, t, \zeta), \\
& \left(\frac{\left[G_{1}\right]_{1}^{L_{1} \cup L_{3}}(x, t, \zeta)}{y(\zeta)},\left[G_{3}\right]_{2}^{L_{1} \cup L_{2} \cup L_{3} \cup L_{4}(x, t, \zeta)}\right) \\
& =\left(\left[G_{3}\right]_{1}^{L_{5} \cup L_{6} \cup L_{7} \cup L_{8}}(x, t, \zeta), \frac{\left[G_{1}\right]_{2}^{L_{6} \cup L_{8}}(x, t, \zeta)}{\overline{y(\bar{\zeta})}}\right) Q_{4}(x, t, \zeta) .
\end{aligned}
$$

Therefore, from the Eqs. (2.34a)-(2.34d), we can get that the jump matrices $\left\{Q_{i}(x, t, \zeta)\right\}_{1}^{4}$ satisfy the Eq. (2.30).

Assumption 2.4 Assume that the zeros of $\rho(\zeta)$ and $y(\zeta)$ satisfy the assumptions as follows

- The spectral function $y(\zeta)$ has $2 n$ simple zeros $\left\{\varsigma_{j}\right\}_{j=1}^{2 n}, 2 n=2 n_{1}+2 n_{2}$, if $\left\{\varsigma_{j}\right\}_{1}^{2 n_{1}} \in L_{1} \cup L_{3}$, then $\left\{\bar{\zeta}_{j}\right\}_{1}^{2 n_{2}} \in L_{8} \cup L_{6}$.

- The spectral function $\rho(\zeta)$ has $2 N$ simple zeros $\left\{\eta_{j}\right\}_{j=1}^{2 N}, 2 N=2 N_{1}+2 N_{2}$, if $\left\{\eta_{j}\right\}_{1}^{2 N_{1}} \in L_{5} \cup L_{7}$, then $\left\{\bar{\eta}_{j}\right\}_{1}^{2 N_{2}} \in L_{4} \cup L_{2}$.

- The spectral function $y(\zeta)$ and $\rho(\zeta)$ have no common zeros.

Proposition 2.5 The matrix function $D(x, t, \zeta)$ defined by Eqs. $(2.27 a)-(2.27 d)$ meets the following residue conditions:

$$
\begin{gathered}
\operatorname{Res}\left\{[D(x, t, \zeta)]_{1}, \varsigma_{j}\right\}=\frac{1}{z\left(\varsigma_{j}\right) \dot{y}\left(\varsigma_{j}\right)} e^{2 i \omega\left(\varsigma_{j}\right)}\left[D\left(x, t, \varsigma_{j}\right)\right]_{2}, j=1, \ldots, 2 n_{1}, \\
\operatorname{Res}\left\{[D(x, t, \zeta)]_{2}, \bar{\varsigma}_{j}\right\}=-\frac{1}{\overline{z\left(\varsigma_{j}\right) \dot{y}\left(\varsigma_{j}\right)}} e^{-2 i \omega\left(\bar{\varsigma}_{j}\right)}\left[D\left(x, t, \bar{\zeta}_{j}\right)\right]_{1}, j=1, \ldots, 2 n_{2},
\end{gathered}
$$




$$
\begin{aligned}
& \operatorname{Res}\left\{[D(x, t, \zeta)]_{1}, \eta_{j}\right\}=-\frac{\overline{Z\left(\bar{\eta}_{j}\right)}}{y\left(\eta_{j}\right) \dot{\rho}\left(\eta_{j}\right)} e^{2 i \omega\left(\eta_{j}\right)}\left[D\left(x, t, \eta_{j}\right)\right]_{1}, j=1, \ldots, 2 N_{1}, \\
& \operatorname{Res}\left\{[D(x, t, \zeta)]_{2}, \bar{\eta}_{j}\right\}=\frac{Z\left(\bar{\eta}_{j}\right)}{\overline{y\left(\eta_{j}\right) \dot{\rho}\left(\eta_{j}\right)}} e^{-2 i \omega\left(\bar{\eta}_{j}\right)}\left[D\left(x, t, \bar{\eta}_{j}\right)\right]_{2}, j=1, \ldots, 2 N_{2},
\end{aligned}
$$

where $\dot{\rho}(\zeta)=\frac{d \rho}{d \zeta}$.

Proof We only verify the residue relationship Eq. (2.35a) as follows:

Due to $D(x, t, \zeta)=\left(\frac{\left[G_{1}\right]_{1}^{L_{1} \cup L_{3}}}{y\left(\zeta \zeta_{2} L_{3}\right.},\left[G_{3}\right]_{2}^{L_{1} \cup L_{2} \cup L_{3} \cup L_{4}}\right)$, which means that the zeros $\left\{\varsigma_{j}\right\}_{1}^{2 n_{1}}$ of $y(\zeta)$ are the poles of $\frac{\left[G_{1}\right]_{1}^{1} L_{L} L_{3}}{y(\zeta)}$. Then, we have

$$
\operatorname{Res}\left\{\frac{G_{1}^{L_{1} \cup L_{3}}(x, t, \zeta)}{y(\zeta)}, \varsigma_{j}\right\}=\lim _{\zeta \rightarrow \varsigma_{j}}\left(\zeta-\varsigma_{j}\right) \frac{\left[G_{1}\right]_{1}^{L_{1} \cup L_{3}}(x, t, \zeta)}{y(\zeta)}=\frac{\left[G_{1}\right]_{1}^{L_{1} \cup L_{3}}\left(x, t, \varsigma_{j}\right)}{\dot{y}\left(\varsigma_{j}\right)} .
$$

Taking $\zeta=\varsigma_{j}$ into the second equation of Eqs. (2.33a)-(2.33b) yields

$$
\left[G_{1}\right]_{1}^{L_{1} \cup L_{3}}\left(x, t, \varsigma_{j}\right)=\frac{1}{y\left(\varsigma_{j}\right)} e^{2 i \omega\left(\varepsilon_{j}\right)}\left[G_{3}\right]_{2}^{L_{1} \cup L_{2} \cup L_{3} \cup L_{4}}\left(x, t, \varsigma_{j}\right) .
$$

According to the Eqs. (2.36) and (2.37), we get

$$
\operatorname{Res}\left\{\frac{\left[G_{1}\right]_{1}^{L_{1} \cup L_{3}}(x, t, \zeta)}{y(\zeta)}, \varsigma_{j}\right\}=\frac{1}{z\left(\varsigma_{j}\right) \dot{y}\left(\varsigma_{j}\right)} e^{2 i \omega\left(\varsigma_{j}\right)}\left[G_{3}\right]_{2}^{L_{1} \cup L_{2} \cup L_{3} \cup L_{4}}\left(x, t, \varsigma_{j}\right)
$$

Therefore, the Eq. (2.38) leads to the Eq. (2.35a), and the remaining three residue relationships Eqs. (2.35b)-(2.35d) can be similarly proved.

\subsection{The Global Relation}

In this subsection, we give the spectral functions which are not independent but satisfy a nice global relation. In fact, at the boundary of the region $(\xi, \tau): 0<\xi<\infty, 0<\tau<t$, the integral of the one-form $F(x, t, \zeta)$ given by Eq.(2.7) vanishes. Let $G(x, t, \zeta)=G_{3}(x, t, \zeta)$, we get 


$$
\begin{gathered}
\int_{\infty}^{0} e^{i \zeta \xi \hat{\Lambda}}\left(P G_{3}\right)(\xi, 0, \zeta) d \xi+\int_{0}^{t} e^{-i\left(8 \gamma \zeta^{4}-2 \zeta^{2}\right) \tau \hat{\Lambda}}\left(R G_{3}\right)(0, \tau, \zeta) d \tau \\
\quad+e^{-i\left(8 \gamma \zeta^{4}-2 \zeta^{2}\right) t \hat{\Lambda}} \times \int_{0}^{\infty} e^{i \zeta \xi \hat{\Lambda}}\left(P G_{3}\right)(\xi, t, \zeta) d \xi \\
=\lim _{x \rightarrow \infty} e^{i \zeta x \hat{\Lambda}} \int_{0}^{t} e^{-i\left(8 \gamma \zeta^{4}-2 \zeta^{2}\right) \tau \hat{\Lambda}}\left(R G_{3}\right)(x, \tau, \zeta) d \tau .
\end{gathered}
$$

On the one hand, according to the definition of $\psi(\zeta)$ in Eq. (2.15), together with the Eq. (2.20b), we can get the first term of the Eq. (2.39)

$$
\psi(\zeta)-I \text {. }
$$

Let $x=0$ in the Eq. (2.16), we get

$$
G_{3}(0, \tau, \zeta)=G_{1}(0, \tau, \zeta) e^{i\left(8 \gamma \zeta^{4}-2 \zeta^{2}\right) \tau \hat{\Lambda}} \psi(\zeta),
$$

therefore

$$
e^{-i\left(8 \gamma \zeta^{4}-2 \zeta^{2}\right) \tau \hat{\Lambda}}\left(R G_{3}\right)(0, \tau, \zeta)=\left[e^{-i\left(8 \gamma \zeta^{4}-2 \zeta^{2}\right) \tau \hat{\Lambda}}\left(R G_{1}\right)(0, \tau, \zeta)\right] \psi(\zeta) .
$$

On the other hand, the Eqs. (2.41) and (2.19a) mean that the second term of the Eq. (2.39) is

$$
\int_{0}^{t} e^{-i\left(8 \gamma \zeta^{4}-2 \zeta^{2}\right) \tau \hat{\Lambda}}\left(R G_{3}\right)(0, \tau, \zeta) d \tau=\left[e^{-i\left(8 \gamma \zeta^{4}-2 \zeta^{2}\right) t \hat{\Lambda}} R G_{1}(0, t, \zeta)-I\right] \psi(\zeta) .
$$

For $x \rightarrow \infty, u(x, t) \in \mathscr{S}$, the Eq. (2.39) is equivalent to

$$
\phi^{-1}(t, \zeta) \psi(\zeta)+e^{-i\left(8 \gamma \zeta^{4}-2 \zeta^{2}\right) t \hat{\Lambda}} \times \int_{0}^{\infty} e^{i \zeta \xi \hat{\Lambda}}\left(P G_{3}\right)(\xi, t, \zeta) d \xi=I,
$$

where the first column of the Eq. (2.42) is valid for $\zeta \in L_{5} \cup L_{6} \cup L_{7} \cup L_{8}$ and the second column of the Eq. (2.42) is valid for $\zeta \in L_{1} \cup L_{2} \cup L_{3} \cup L_{4}$, and $\phi(t, \zeta)$ is given by

$$
\phi^{-1}(t, \zeta)=e^{-i\left(8 \gamma \zeta^{4}-2 \zeta^{2}\right) t \hat{\Lambda}} G_{1}(0, t, \zeta)
$$

Owing to $\phi(\zeta)=\phi(T, \zeta)$ and let $t=T$, then, the Eq. (2.42) is equivalent to

$$
\phi^{-1}(\zeta) \psi(\zeta)+e^{-i\left(8 \gamma \zeta^{4}-2 \zeta^{2}\right) T \hat{\Lambda}} \times \int_{0}^{\infty} e^{i \zeta \xi \hat{\Lambda}}\left(P G_{3}\right)(\xi, T, \zeta) d \xi=I .
$$

Hence, the 1,2 component of the Eq. (2.43) equals

$$
y(\zeta) Z(\zeta)-Y(\zeta) z(\zeta)=e^{-2 i\left(8 \gamma \zeta^{4}-2 \zeta^{2}\right) T} E(\zeta),
$$

where $E(\zeta)$ is expressed as 


$$
E(\zeta)=\int_{0}^{\infty} e^{i \zeta \xi}\left(P G_{3}\right)_{12}(\xi, T, \zeta) d \xi
$$

which is the so-called global relation.

\section{The Spectral Functions}

Definition 3.1 (Related to $y(\zeta), z(\zeta))$ Let $u_{0}(x)=u(x, 0) \in \mathscr{S}$, the mapping

$$
\mathrm{H}_{1}:\left\{u_{0}(x)\right\} \rightarrow\{y(\zeta), z(\zeta)\},
$$

is defined by

$$
\left(\begin{array}{l}
z(\zeta) \\
y(\zeta)
\end{array}\right)=\left[G_{3}\right]_{2}^{L_{1} \cup L_{2} \cup L_{3} \cup L_{4}}(0 ; \zeta)=\left(\begin{array}{c}
\left(G_{3}\right)_{12}^{L_{1} \cup L_{2} \cup L_{3} \cup L_{4}}(0 ; \zeta) \\
\left(G_{3}\right)_{22}^{L_{1} \cup L_{2} \cup L_{3} \cup L_{4}}(0 ; \zeta)
\end{array}\right), \operatorname{Im} \zeta \geq 0
$$

where $G_{3}(x, \zeta)$ and $P(x, \zeta)$ are given by Eqs. (2.20b) and (2.21), respectively.

Proposition 3.2 The $y(\zeta)$ and $z(\zeta)$ satisfy the properties as follows

(i) For $\operatorname{Im} \zeta<0, y(\zeta)$ and $z(\zeta)$ are analytic functions,

(ii) $y(\zeta)=1+O\left(\frac{1}{\zeta}\right), z(\zeta)=O\left(\frac{1}{\zeta}\right)$, as $\zeta \rightarrow \infty$,

(iii) $\operatorname{For} \zeta \in \mathrm{R}, \operatorname{det} \psi(\zeta)=|y(\zeta)|^{2}+|z(\zeta)|^{2}=1$,

(iv) $\mathrm{S}_{1}=\mathrm{H}_{1}^{-1}:\{y(\zeta), z(\zeta)\} \rightarrow\left\{u_{0}(x)\right\} u_{0}(x)$ has the following form

$$
u_{0}(x)=2 i \lim _{\zeta \rightarrow \infty}\left(\zeta D^{(x)}(x, \zeta)\right)_{12},
$$

where $D^{(x)}(x, \zeta)$ meets the following RH problem.

Remark 3.3 Assume that

$$
\begin{aligned}
& D_{+}^{(x)}(x, \zeta)=\left(\frac{\left[G_{1}\right]_{1}^{L_{1} \cup L_{2} \cup L_{3} \cup L_{4}}(x, \zeta)}{y(\zeta)},\left[G_{3}\right]_{2}^{L_{1} \cup L_{2} \cup L_{3} \cup L_{4}}(x, \zeta)\right), \operatorname{Im} \zeta \geq 0, \\
& D_{-}^{(x)}(x, \zeta)=\left(\left[G_{3}\right]_{1}^{L_{5} \cup L_{6} \cup L_{7} \cup L_{8}}(x, \zeta), \frac{\left[G_{1}\right]_{2}^{L_{5} \cup L_{6} \cup L_{7} \cup L_{8}}(x, \zeta)}{\overline{y(\bar{\zeta})}}\right), \operatorname{Im} \zeta \leq 0,
\end{aligned}
$$

hence, $D^{(x)}(x, \zeta)$ admits the RH problem

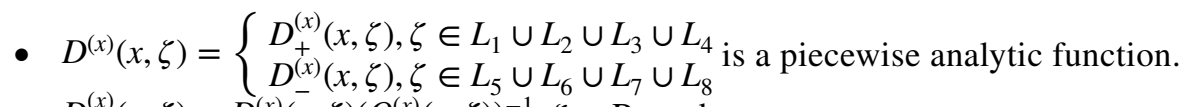

- $D_{+}^{(x)}(x, \zeta)=D_{-}^{(x)}(\bar{x}, \zeta)\left(Q^{(x)}(x, \zeta)\right)^{-1}, \zeta \in \mathrm{R}$, and 


$$
Q^{(x)}(x, \zeta)=\left(\begin{array}{cc}
\frac{1}{y(\zeta) \overline{y(\bar{\zeta})}} & \frac{z(\zeta)}{\overline{y(\bar{\zeta})}} e^{-2 i \zeta x} \\
-\frac{z(\bar{\zeta})}{y(\zeta)} e^{2 i \zeta x} & 1
\end{array}\right)
$$

- $D^{(x)}(x, \zeta) \rightarrow \mathrm{I}, \zeta \rightarrow \infty$.

- $y(\zeta)$ possesses $2 n$ simple zeros $\left\{\varsigma_{j}\right\}_{1}^{2 n}, 2 n=2 n_{1}+2 n_{2}$, assume that $\left\{\varsigma_{j}\right\}_{1}^{2 n_{1}}$ are parts of $L_{1} \cup L_{2} \cup L_{3} \cup L_{4}$, then, $\left\{\bar{\zeta}_{j}\right\}_{1}^{2 n_{2}}$ are parts of $L_{5} \cup L_{6} \cup L_{7} \cup L_{8}$.

- $\left[D_{+}^{(x)}\right]_{1}(x, \zeta)$ enjoys simple poles for $\zeta=\left\{\varsigma_{j}\right\}_{1}^{2 n_{1}}$ and the $\left[D_{-}^{(x)}\right]_{2}(x, \zeta)$ has simple poles for $\zeta=\left\{\bar{\zeta}_{j}\right\}_{1}^{2 n_{2}}$. In this case, the residue relations are define by

$$
\begin{aligned}
& \operatorname{Res}\left\{\left[D^{(x)}(x, \zeta)\right]_{1}, \varsigma_{j}\right\}=\frac{e^{2 i \varsigma_{j} x}}{z\left(\varsigma_{j}\right) \dot{y}\left(\varsigma_{j}\right)}\left[D^{(x)}\left(x, \varsigma_{j}\right)\right]_{2}, \\
& \operatorname{Res}\left\{\left[D^{(x)}(x, \zeta)\right]_{2}, \bar{\varsigma}_{j}\right\}=\frac{e^{-2 i \bar{\zeta}_{j} x}}{\overline{z\left(\varsigma_{j}\right) \dot{y}\left(\varsigma_{j}\right)}}\left[D^{(y)}\left(x, \bar{\varsigma}_{j}\right)\right]_{1} .
\end{aligned}
$$

Definition 3.4 (Related to $Y(\zeta), Z(\zeta)$ ). Let $v_{0}(t), v_{1}(t), v_{2}(t), v_{3}(t) \in \mathscr{S}$, the mapping

$$
\mathrm{H}_{2}:\left\{v_{0}(t), v_{1}(t), v_{2}(t), v_{3}(t)\right\} \rightarrow\{Y(\zeta), Z(\zeta)\},
$$

is defined by

$$
\left(\begin{array}{c}
Y(\zeta) \\
Z(\zeta)
\end{array}\right)=\left[G_{2}\right]_{2}{ }^{L_{2} \cup L_{4} \cup L_{6} \cup L_{8}}(t, \zeta)=\left(\begin{array}{c}
\left(G_{2}\right)_{12}^{L_{2} \cup L_{4} \cup L_{6} \cup L_{8}}(t, \zeta) \\
\left(G_{2}\right)_{22}^{L_{2} \cup L_{4} \cup L_{6} \cup L_{8}}(t, \zeta)
\end{array}\right)
$$

where $G_{2}(t, \zeta)$ and $R(t, \zeta)$ are given by Eqs. (2.19b) and (2.21), respectively.

Proposition 3.5 The $Y(\zeta)$ and $Z(\zeta)$ satisfy the properties as follows

(i) For $\operatorname{Im}\left(8 \gamma \zeta^{4}-2 \zeta^{2}\right) \leq 0, Y(\zeta), Z(\zeta)$ are analytic functions,

(ii) $Y(\zeta)=1+O\left(\frac{1}{\zeta}\right)+O\left(\frac{e^{-2 i\left(8 \gamma \zeta^{4}-2 \zeta^{2}\right) T}}{\zeta}\right), Z(\zeta)=O\left(\frac{1}{\zeta}\right)+O\left(\frac{e^{-2 i\left(8 \gamma \zeta^{4}-2 \zeta^{2}\right) T}}{\zeta}\right)$, as $\zeta \rightarrow \infty$,

(iii) $F o r \zeta \in \mathrm{C}, \operatorname{det} \phi(\zeta)=Y(\zeta) \overrightarrow{Y(\bar{\zeta})}+Z(\zeta) \overline{Z(\bar{\zeta})}=1,\left(\left(8 \gamma \zeta^{4}-2 \zeta^{2}\right) \in \mathrm{R}\right.$, if $\left.T=\infty\right)$,

(iv) $\mathrm{S}_{2}=\mathrm{H}_{2}^{-1}:\{Y(\zeta), Z(\zeta)\} \rightarrow\left\{v_{0}(t), v_{1}(t), v_{2}(t), v_{3}(t)\right\}$

$$
v_{0}(t)=2 i \lim _{\zeta \rightarrow \infty}\left(\zeta D^{(t)}(t, \zeta)\right)_{12},
$$

$v_{1}(t)=\lim _{\zeta \rightarrow \infty}\left[4\left(\zeta^{2} D^{(t)}(t, \zeta)\right)_{12}+2 i v_{0}(t)\left(\zeta D^{(t)}(t, \zeta)\right)_{22}\right]$, 


$$
\begin{aligned}
\gamma v_{2}(t)= & \lim _{\zeta \rightarrow \infty}\left[-8 i \gamma\left(\zeta^{3} D^{(t)}(t, \zeta)\right)_{12}+4 \gamma v_{0}(t)\left(\zeta^{2} D^{(t)}(t, \zeta)\right)_{22}\right. \\
& +2 i \gamma\left(v_{0}^{2}(t)\left(\zeta D^{(t)}(t, \zeta)\right)_{12}+v_{1}(t)\left(\zeta D^{(t)}(t, \zeta)\right)_{22}\right) \\
& \left.+2 i\left(\zeta D^{(t)}(t, \zeta)\right)_{12}-\left(2 \gamma v_{0}^{2}(t)+1\right) v_{0}(t)\right] \\
\gamma v_{3}(t)= & \lim _{\zeta \rightarrow \infty}\left\{-16 \gamma\left(\zeta^{4} D^{(t)}(t, \zeta)\right)_{12}+4\left(\zeta^{2} D^{(t)}(t, \zeta)\right)_{12}\right. \\
& -8 i \gamma v_{0}(t)\left(\zeta^{3} D^{(t)}(t, \zeta)\right)_{22}+4 \gamma\left(v_{0}^{2}(t)\left(\zeta^{2} D^{(t)}(t, \zeta)_{12}+v_{1}(t)\left(\zeta^{2} D^{(t)}(t, \zeta)\right)_{22}\right)\right. \\
& +i\left(6 \gamma v_{0}^{2}(t)+1\right) v_{1}(t)-2 i\left[\gamma\left(v_{0}(t) \overline{v_{1}}(t)-\bar{v}_{0}(t) v_{1}(t)\right)\left(\zeta D^{(t)}(t, \zeta)\right)_{12}\right. \\
& \left.\left.-\left(\gamma v_{2}(t)+\left(2 \gamma v_{0}^{2}(t)+1\right) v_{0}(t)\right)\left(\zeta D^{(t)}(t, \zeta)\right)_{22}\right]\right\}
\end{aligned}
$$

where $D^{(1)}(t), D^{(2)}(t), D^{(3)}(t), D^{(4)}(t)$ meet the following asymptotic expansion

$$
\begin{aligned}
& D^{(t)}(t, \zeta)= \mathrm{I} \\
&+\frac{D^{(1)}(t, \zeta)}{\zeta}+\frac{D^{(2)}(t, \zeta)}{\zeta^{2}}+\frac{D^{(3)}(t, \zeta)}{\zeta^{3}}+\frac{D^{(4)}(t, \zeta)}{\zeta^{4}} \\
&+O\left(\frac{1}{\zeta^{5}}\right), \text { as } \zeta \rightarrow \infty
\end{aligned}
$$

and $D^{(t)}(t, \zeta)$ meets the following RH problem.

Remark 3.6 Assume that

$$
\begin{gathered}
D_{+}^{(t)}(t, \zeta)=\left(\frac{\left[G_{1}\right]_{1}^{L_{1} \cup L_{3} \cup L_{5} \cup L_{7}}(t, \zeta)}{Y(\zeta)},\left[G_{3}\right]_{2}^{L_{1} \cup L_{3}}(t, \zeta)\right), \operatorname{Im}\left(8 \gamma \zeta^{4}-2 \zeta^{2}\right) \geq 0 \\
D_{-}^{(t)}(t, \zeta)=\left(\left[G_{3}\right]_{1}^{L_{2} \cup L_{4}}(t, \zeta), \frac{\left[G_{1}\right]_{2}^{L_{2} \cup L_{4}}(t, \zeta)}{\overline{Y(\bar{\zeta})}}\right), \operatorname{Im}\left(8 \gamma \zeta^{4}-2 \zeta^{2}\right) \leq 0
\end{gathered}
$$

hence, $D^{(t)}(t, \zeta)$ admits the RH problem as follows.

- $D^{(t)}(t, \zeta)=\left\{\begin{array}{l}D_{+}^{(t)}(t, \zeta), \zeta \in L_{1} \cup L_{4} \cup L_{5} \cup L_{8} \\ D_{-}^{(t)}(t, \zeta), \zeta \in L_{2} \cup L_{3} \cup L_{6} \cup L_{7}\end{array}\right.$ is a piecewise analytic function.

- $D_{+}^{(t)}(t, \zeta)=D_{-}^{(t)}(t, \zeta) Q^{(t)}(t, \zeta), \zeta \in \Gamma_{m}, m=1,2,3,4$, and

$$
Q^{(t)}(t, \zeta)=\left(\begin{array}{cc}
1 & -\frac{Z(\zeta)}{\overline{Y(\bar{\zeta})}} e^{2 i\left(8 \gamma \zeta^{4}-2 \zeta^{2}\right) t} \\
-\frac{\overline{Z(\bar{\zeta})}}{Y(\zeta)} e^{-2 i\left(8 \gamma \zeta^{4}-2 \zeta^{2}\right) t} & \frac{1}{Y(\zeta) \overline{Y(\bar{\zeta})}}
\end{array}\right)
$$

- $D^{(t)}(t, \zeta) \rightarrow \mathrm{I}, \zeta \rightarrow \infty$

- $Y(\zeta)$ possesses $2 k$ simple zeros $\left\{v_{j}\right\}_{1}^{2 k}, 2 k=2 k_{1}+2 k_{2}$, assume that $\left\{v_{j}\right\}_{1}^{2 k_{1}}$ are parts of $L_{1} \cup L_{3}$, then, $\left\{\bar{v}_{j}\right\}_{1}^{2 k_{2}}$ are parts of $L_{2} \cup L_{4}$.

- $\left[D_{+}^{(t)}\right]_{1}(t, \zeta)$ enjoys simple poles for $\zeta=\left\{v_{j}\right\}_{1}^{2 k_{1}}$ and the $\left[D_{-}^{(t)}\right]_{2}(t, \Lambda)$ has simple poles for $\zeta=\left\{\bar{v}_{j}\right\}_{1}^{2 k_{2}}$. In this case, the residue relations are defined by 


$$
\begin{aligned}
& \operatorname{Res}\left\{\left[D^{(t)}(t, \zeta)\right]_{1}, v_{j}\right\}=\frac{e^{-2\left(8 \gamma v_{j}^{4}-2 v_{j}^{2}\right) t}}{Z\left(v_{j}\right) \dot{Y}\left(v_{j}\right)}\left[D^{(t)}\left(t, v_{j}\right)\right]_{2}, \\
& \operatorname{Res}\left\{\left[D^{(t)}(t, \zeta)\right]_{2}, \bar{v}_{j}\right\}=\frac{e^{2\left(8 \gamma \bar{v}_{j}^{4}-2 \bar{v}_{j}^{2}\right) t}}{\overline{Z\left(\bar{v}_{j}\right)} \overline{\dot{Y}\left(v_{j}\right)}}\left[D^{(t)}\left(t, v_{j}\right)\right]_{1} .
\end{aligned}
$$

\section{The Riemann-Hilbert Problem}

In this part, we give two important results in theorem form.

Theorem 4.1 Let $u_{0}(x) \in \mathscr{S}\left(\mathrm{R}^{+}\right)$, and $\Psi(\zeta), \phi(\zeta)$ defined in terms of $y(\zeta), z(\zeta)$, $Y(\zeta), Z(\zeta)$ are showed in Eq. (2.23). And the $y(\zeta), z(\zeta), Y(\zeta), Z(\zeta)$ denoted by functions $u_{0}(x), v_{j}(t), j=0, \ldots, 3$ are showed in Definitions 3.1 and 3.2. Assume that the function $y(\zeta)$ possesses the possible simple zeros $\left\{\varsigma_{j}\right\}_{j=1}^{2 n}$, and the function $\rho(\zeta)$ possesses the possible simple zeros $\left\{\eta_{j}\right\}_{j=1}^{2 N}$. Therefore, the solution of the FODNLS equation (1.2) is

$$
u(x, t)=2 i \lim _{\zeta \rightarrow \infty}(\zeta D(x, t, \zeta))_{12},
$$

where $D(x, t, \zeta)$ is the solution of the $R H$ problems as follows:

- $D(x, t, \zeta)$ is a piecewise analytic function for $\zeta \in \mathrm{C} \backslash \Gamma_{m}(m=1, \ldots, 4)$.

- $D(x, t, \zeta)$ jump appears on the curves $\Gamma_{m}$, which meets the jump conditions as

$$
D_{+}(x, t, \zeta)=D_{-}(x, t, \zeta) Q(x, t, \zeta), \zeta \in \Gamma_{m}, m=1, \ldots, 4 .
$$

- $D(x, t, \zeta)=\mathrm{I}+O\left(\frac{1}{\zeta}\right), \zeta \rightarrow \infty$.

- $D(x, t, \zeta)$ possesses residue relations showed in Proposition 2.3.

Thus, the matrix function $D(x, t, \zeta)$ exists and is unique. Furthermore

$$
\begin{aligned}
u(x, 0) & =u_{0}(x), u(0, t)=v_{0}(t), u_{x}(0, t)=v_{1}(t), \\
u_{x x}(0, t) & =v_{2}(t), u_{x x x}(0, t)=v_{3}(t) .
\end{aligned}
$$

Proof In fact, if we assume that $y(\zeta)$ and $\rho(\zeta)$ have no zeros, then the matrix-valued function $D(x, t, \zeta)$ satisfies a non-sigular $\mathrm{RH}$ problem, and then we prove that this non-sigular RH problem has a unique global solution using the symmetric property of the jump matrix $Q(x, t, \zeta)$ and the scattering matrix. If $y(\zeta)$ and $\rho(\zeta)$ have only a finite number of zeros, we can map this situation to a situation without zeros, which can transform into a system of algebraic equations, this algebraic equations can always be solved uniquely.

Similar Ref. [12], we give the following vanishing theorem without proof. 
Theorem 4.2 (The vanishing theorem) If the matrix function $D(x, t, \zeta) \rightarrow 0(\zeta \rightarrow \infty)$, then, the RH problem in Theorem 4.1 possesses only the zero solution.

\section{Conclusions}

In this paper, we use the Fokas method to construct the RH problem of the FODNLS equation on the half-line. When the parameter $\gamma=0$, it can be reduced to the RH problem of the classical nonlinear Schrödinger equation on the half-line. We introduced important functions for spectral analysis of the Lax pair, established the basic RH problem, and gave the global relationship between spectral functions. Furthermore, we can analyze the integrable FODNLS equation (1.2) on a finite interval, and also discuss the asymptotic behavior for the solution of the integrable FODNLS equation (1.2). These two questions will be studied in our future investigation.

Acknowledgements We are thankful to the reviewers for the careful reading and suggestions that improved the manuscript. The work is supported by National Natural Science Foundation of China (Grant Nos. 11805114, 11975145). Natural Science Foundation of Anhui Province (Grant No. 2108085QA09). Postdoctoral Fund of Zhejiang Normal University (Grant No. ZC304021909).

Open Access This article is distributed under the terms of the Creative Commons Attribution 4.0 International License (http://creativecommons.org/licenses/by/4.0/), which permits unrestricted use, distribution, and reproduction in any medium, provided you give appropriate credit to the original author(s) and the source, provide a link to the Creative Commons license, and indicate if changes were made.

Author Contributions $\mathrm{BH}$ and $\mathrm{LZ}$ formally analyzes and write original draft, QL write the manuscript, $\mathrm{BH}, \mathrm{LZ}$ and $\mathrm{NZ}$ are in charge of funding acquisition.

Data Availability Statement The authors confirm that the data supporting the findings of this study are available within the article or its supplementary materials.

\section{Declarations}

Conflict of interest The authors declare they have no conflicts of interest.

Open Access This article is licensed under a Creative Commons Attribution 4.0 International License, which permits use, sharing, adaptation, distribution and reproduction in any medium or format, as long as you give appropriate credit to the original author(s) and the source, provide a link to the Creative Commons licence, and indicate if changes were made. The images or other third party material in this article are included in the article's Creative Commons licence, unless indicated otherwise in a credit line to the material. If material is not included in the article's Creative Commons licence and your intended use is not permitted by statutory regulation or exceeds the permitted use, you will need to obtain permission directly from the copyright holder. To view a copy of this licence, visit http://creativecommons.org/licen ses/by/4.0/.

\section{References}

1. Gardner, C.S., Green, J.M., Kruskal, M.D., Miüra, R.M.: Method for solving the Korteweg-de Vries equation. Phys. Rev. Lett. 19(19), 1095-1097 (1967) 
2. Hirota, R.: Exact solution of the Korteweg-de Vries equation for multiple collisions of solitons. Phys. Rev. Lett. 27(18), 1192-1194 (1971)

3. Wahlquist, H.D., Estabrook, F.B.: Bäcklund transformation for solutions of the Korteweg-de Vries equation. Phys. Rev. Lett. 31(23), 1386-1390 (1973)

4. Matveev, V.B., Salle, M.A.: Darboux Transformations and Solitons. Springer, Berlin (1991)

5. Feng, Z.S., Wang, X.H.: Explicit exact solitary wave solutions for the Kundu equation and the derivative Schrödinger equation. Phys. Scr. 64, 7-14 (2001)

6. Fokas, A.S.: A unified transform method for solving linear and certain nonlinear PDEs. Proc. R. Soc. Lond. A 453, 1411-1443 (1997)

7. Fokas, A.S.: Integrable nonlinear evolution equations on the half-line. Commun. Math. Phys. 230(1), 1-39 (2002)

8. Fokas, A.S., Its, A.R., Sung, L.Y.: The nonlinear Schrödinger equation on the half-line. Nonlinearity 18, 1771-1822 (2005)

9. Lenells, J.: The derivative nonlinear Schrödinger equation on the half-line. Phys. D 237(23), 30083019 (2008)

10. Lenells, J.: Boundary value problems for the stationary axisymmetric Einstein equations: a disk rotating around a black hole. Commun. Math. Phys. 304, 585-635 (2011)

11. Xia, B.Q., Fokas, A.S.: Initial-boundary value problems associated with the Ablowitz-Ladik system. Phys. D 364, 27-61 (2018)

12. Hu, B.B., Xia, T.C.: A Riemann-Hilbert approach to the initial-boundary value problem for KunduEckhaus equation on the half line. Complex Var. Elliptic Equ. 64(12), 2019-2039 (2019)

13. Chen, S.Y., Yan, Z.Y.: The Hirota equation: Darboux transform of the Riemann-Hilbert problem and higher-order rogue waves. Appl. Math. Lett. 95, 65-71 (2019)

14. Huang, L.: The initial-boundary-value problems for the Hirota equation on the half-line. Chin. Ann. Math. 41(1), 117-132 (2020)

15. Pu, X.K., Guo, B.L.: Initial boundary value problem for the $3 \mathrm{D}$ magnetic-curvature-driven Rayleigh-Taylor model. Acta Math. Sci. 40(2), 529-542 (2020)

16. Hu, B.B., Zhang, L., Xia, T.C., Zhang, N.: On the Riemann-Hilbert problem of the Kundu equation. Appl. Math. Comput. 381, 125262 (2020)

17. Hu, B.B., Zhang, L., Xia, T.C.: On the Riemann-Hilbert problem of a generalized derivative nonlinear Schrödinger equation. Commun. Theor. Phys. 73, 015002 (2021)

18. Hu, B.B., Zhang, L., Zhang, N.: On the Riemann-Hilbert problem for the mixed Chen-Lee-Liu derivative nonlinear Schrödinger equation. J. Comput. Appl. Math. 390, 113393 (2021)

19. Lenells, J.: Initial-boundary value problems for integrable evolution equations with $3 \times 3$ Lax pairs. Phys. D 241, 857-875 (2012)

20. Lenells, J.: The Degasperis-Procesi equation on the half-line. Nonlinear Anal. 76, 122-139 (2013)

21. Anne, B.D.M., Shepelsky, D.: A Riemann-Hilbert approach for the Degasperis-Procesi equation. Nonlinearity 26(7), 2081-2107 (2013)

22. $\mathrm{Xu}, \mathrm{J} .:$ Initial-boundary value problem for the two-component nonlinear Schrödinger equation on the half-line. J. Nonlinear Math. Phys. 23, 167-189 (2016)

23. Liu, H., Geng, X.G.: Initial-boundary problems for the vector modified Korteweg-deVries equation via Fokas unified transform method. J. Math. Anal. Appl 440(2), 578-596 (2016)

24. Tian, S.F.: Initial-boundary value problems for the general coupled nonlinear Schrödinger equation on the interval via the Fokas method. J. Differ. Equ. 262, 506-558 (2017)

25. Yan, Z.Y.: Initial-boundary value problem for an integrable spin-1 Gross-Pitaevskii system with a $4 \times 4$ Lax pair on a finite interval. J. Math. Phys. 60(8), 1-70 (2019)

26. Zhu, Q.Z., Xu, J., Fan, E.G.: Initial-boundary value problem for two-component Gerdjikov-Ivanov equation on the interval. J. Nonlinear Math. Phys. 25, 136-165 (2018)

27. Zhang, H.Q., Pei, Z.J., Ma, W.X.: Riemann-Hilbert approach for a coherently-coupled nonlinear Schrödinger system associated with a $4 \times 4$ matrix spectral problem. Chaos Solitons Fractals $\mathbf{1 2 3}$, 429-434 (2019)

28. Hu, B.B., Xia, T.C., Zhang, N.: The unified transform method to initial-boundary value problem of the coupled cubic-quintic nonlinear Schrödinger system. Complex Anal. Oper. Theory 13(3), 11431159 (2019)

29. Deift, P.A., Zhou, X.: A steepest descent method for oscillatory Riemann-Hilbert problems. Ann. Math. 137, 295-368 (1993)

30. Ma, W.X.: Long-time asymptotics of a three-component coupled mKdV system. Mathematics 7(7), $573(2019)$ 
31. Ma, W.X.: Long-time asymptotics of a three-component coupled nonlinear Schrödinger system. J. Geom. Phys. 153, 103669 (2020)

32. Porsezian, K., Daniel, M., Lakshmanan, M.: On the integrability aspects of the one-dimensional classical continuum isotropic biquadratic Heisenberg spin chain. J. Math. Phys. 33(5), 1807-1816 (1992)

33. Choudhuri, A., Porsezian, K.: Higher-order nonlinear Schrödinger equation with derivative nonKerr nonlinear terms: a model for sub-10-fs-pulse propagation. Phys. Rev. A 88(88), 169 (2013)

34. Azzouzi, F., Triki, H., Mezghiche, K., Akrmi, A.E.: Solitary wave solutions for high dispersive cubic-quintic nonlinear Schrödinger equation. Chaos Solitons Fractals 39(3), 1304-1307 (2009)

35. Daniel, M., Latha, M.M.: A generalized Davydov soliton model for energy transfer in alpha helical proteins. Phys. A 298(3-4), 351-370 (2001)

36. Zhang, H.Q., Tian, B., Meng, X.H., Lü, X., Liu, W.J.: Conservation laws, soliton solutions and modulational instability for the higher-order dispersive nonlinear Schrödinger equation. Eur. Phys. J. B 72(2), 233-239 (2009)

37. Wang, X.L., Zhang, W.G., Zhai, B.G., Zhang, H.Q.: Rogue waves of the higher-order dispersive nonlinear Schrödinger equation. Commun. Theor. Phys. 58(10), 531-538 (2012)

38. Wang, L.H., Porsezian, K., He, J.S.: Breather and rogue wave solutions of a generalized nonlinear Schrödinger equation. Phys. Rev. E 87, 053202 (2013)

39. Yang, B., Zhang, W.G., Zhang, H.Q., Pei, S.B.: Generalized Darboux transformation and rogue wave solutions for the higher-order dispersive nonlinear Schrödinger equation. Phys. Scr. 88(6), $065004(2013)$

40. Liu, W.H., Liu, Y., Zhang, Y.F., Shi, D.D.: Riemann-Hilbert approach for multi-soliton solutions of a fourth-order nonlinear Schrödinger equation. Mod. Phys. Lett. B 33(33), 1950416 (2019)

41. Li, M., Wang, B.T., Xu, T., Shui, J.J.: Study on the generation mechanism of bright and dark solitary waves and rogue wave for a fourth-order dispersive nonlinear Schrödinger equation. Acta Phys. Sin. 69, $010502(2020)$

42. Liu, R.X., Tian, B., Liu, L.C., Qin, B., Lü, X.: Bilinear forms, N-soliton solutions and soliton interactions for a fourth-order dispersive nonlinear Schrödinger equation in condensed-matter physics and biophysics. Phys. B 413, 120-125 (2013)

43. Liu, R.X., Tian, B., Jiang, Y., Wang, P.: Dark solitonic excitations and collisions from a fourthorder dispersive nonlinear Schrödinger model for the alpha helical protein. Commun. Nonlinear Sci. Numer. Simul. 19(3), 520-529 (2014) 